\title{
AUTOPHAGY IN LOAD-INDUCED HEART DISEASE
}

\author{
Hongxin Zhu ${ }^{*}$, Beverly A. Rothermel ${ }^{*}$, and Joseph A. Hill ${ }^{*}$, \\ "Department of Internal Medicine (Cardiology), University of Texas Southwestern Medical Center, \\ Dallas, Texas, USA \\ ${ }^{\dagger}$ Department of Molecular Biology, University of Texas Southwestern Medical Center, Dallas, Texas, \\ USA
}

\begin{abstract}
The heart is a highly plastic organ capable of remodeling in response to changes in physiological or pathological demand. When workload increases, the heart compensates through hypertrophic growth of individual cardiomyocytes to increase cardiac output. However, sustained stress, such as occurs with hypertension or following myocardial infarction, triggers changes in sarcomeric protein composition and energy metabolism, loss of cardiomyocytes, ventricular dilation, reduced pump function, and ultimately heart failure. It has been known for some time that autophagy is active in cardiomyocytes, occurring at increased levels in disease. Yet the potential contribution of cardiomyocyte autophagy to ventricular remodeling and disease pathogenesis has only recently been explored. This latter fact stems largely from the recent emergence of tools to probe molecular mechanisms governing cardiac plasticity and to define the role of autophagic flux in the context of heart disease. In this chapter, we briefly review prominent mouse models useful in the study of loadinduced heart disease and standard techniques used to assess whether a molecular or cellular event is adaptive or maladaptive. We then outline methods available for monitoring autophagic activity in the heart, providing detailed protocols for several techniques unique to working with heart and other striated muscles.
\end{abstract}

\section{INTRODUCTION}

Heart disease is the leading cause of death in the industrialized world. In many instances, heart disease culminates in heart failure, a syndrome characterized by the heart's inability to meet the metabolic demands of the body. Currently, 5 million Americans suffer from chronic heart failure, the leading hospital discharge diagnosis in Medicare (Rosamond et al., 2008). Mortality from heart failure is approximately $50 \%$ at 5 years (Rosamond et al., 2008).

In response to increases in cardiac workload, a compensatory hypertrophic growth response ensues (Hill and Olson, 2008). However, under conditions of sustained load, such as occurs in patients with uncontrolled hypertension, the heart progresses to a state of decompensated heart failure (load-induced heart failure). Consistent with this, it has been shown clinically, epidemiologically, and experimentally that left ventricular hypertrophy is a predictor of increased risk of heart failure (Hein et al., 2003; Levy et al., 1996). As such, recent work has focused on the hypertrophic phenotype itself as a therapeutic target (Frey et al., 2004). However, cellular mechanisms underlying pathological cardiac remodeling remain poorly understood.

Autophagy is a highly conserved mechanism of protein degradation whereby damaged proteins and organelles can be removed and recycled through lysosomes, a process that supports cell survival during times of stress. In the context of certain developmental and disease states, however, autophagy promotes cell death (autophagic programmed cell death, type II PCD) (Levine and Kroemer, 2008; Shintani and Klionsky, 2004). It has been known for 30 years that 
lysosomal pathways are activated in a variety of models of heart disease (Dammrich and Pfeifer, 1983; Decker and Wildenthal, 1980; Decker et al., 1980; Pfeifer et al., 1987; Sybers et al., 1967). Indeed, a number of groups report increases in lysosomal activity in tissue samples from diseased and failing human hearts (Elsasser et al., 2004; Hein et al., 2003; Knaapen et al., 2001; Kostin et al., 2003; Lockshin and Zakeri, 2002; Shimomura et al., 2001; Yamamoto et al., 2000). More recent studies have revealed an important role for autophagy in the cardiomyocyte response to numerous types of stress, and compelling evidence has emerged that this autophagic response participates in the pathogenesis of disease. Recent advances in our understanding of the molecular mechanisms controlling autophagy (Klionsky, 2007;

Kundu and Thompson, 2008; Mizushima, 2007) have led to the emergence of novel tools and techniques to probe this process during development and in the progression of disease. Our laboratory has adapted many of these techniques to monitor autophagy specifically in the heart (Tannous et al., 2008a,b; Zhu et al., 2007), allowing us to explore its contribution to the pathogenesis of load-induced heart failure.

The aim of this chapter is to review standard models of load-induced heart disease, to discuss strategies for assessing whether a molecular event is adaptive or maladaptive, and to outline methods available for monitoring autophagic activity in the heart. As part of this, we provide detailed protocols for several techniques unique to working with heart and other striated muscles.

\section{MOUSE MODELS OF LOAD-INDUCED HEART DISEASE}

\subsection{Thoracic aortic banding (TAB)}

The most commonly used surgical intervention to promote pressure overload-induced cardiac hypertrophy in mice is thoracic aortic banding (TAB; also termed thoracic aortic constriction, TAC). This procedure entails constricting the transverse aortic arch, between the innominate and left common carotid arteries, using a 27-gauge needle as a guide (Rockman et al., 1991). At 3 weeks, the hypertrophic response has reached steady-state (Hill et al., 2000), and the integrity of aortic banding can be confirmed by inspection of the surgical constriction and by visualization of marked differences in caliber of the right and left carotid arteries. In our hands, working with adult C57BL6 mice, this operation induces a stable compensated hypertrophic response with an approximately $40 \%$ increase in the heart weight to body weight ratio at 3 weeks (Hill et al., 2000). The mice display no clinical signs of heart failure or malignant ventricular arrhythmia.

\subsection{Severe thoracic aortic banding (sTAB)}

Tighter constriction of the aorta to the diameter of a 28-gauge needle leads to pressure-overload failure (Rothermel et al., 2005). Mice subjected to STAB gradually develop signs of circulatory failure, including lethargy, impaired mobility, diminished appetite, and peripheral edema. Importantly, sTAB mice have elevated serum levels of tumor necrosis factor (TNF $\alpha$ ), a marker of heart failure (Rothermel et al., 2005). There is a doubling of the heart weight to body weight ratio over the course of three weeks and a substantial increase in mortality. It is important to be aware that, unlike cardiac remodeling in hypertensive patients, the onset of pressure overload is abrupt in the TAB and STAB surgical models. Despite this important difference, these two models have proven to be invaluable techniques for dissecting pathways leading to load-induced hypertrophy and heart failure. 


\section{ANALYSIS OF VENTRICULAR REMODELING}

\subsection{Assessing changes in cardiac function}

Echocardiography is a standard approach for assessing changes in cardiac size and function, such as contractile performance, left ventricular end diastolic dimension (LVEDD), and left ventricular end systolic dimension (LVESD). Percentage fractional shortening, a measure of ventricular contraction, is significantly decreased in STAB hearts, and LVEDD and LVESD are each increased. Technical details will depend on the equipment available; however, many machines designed for use in humans can be adapted for use on mice provided a small enough scan head of sufficient frequency is used. Because anesthesia can depress ventricular function, it is important to carry out these measurements on unanesthetized mice. This can be facilitated by acclimating the mice to handling and using a depilatory agent the day before study to remove chest hair. Invasive measurements of left ventricular pressures, along with estimates of ventricular volume using a conductance catheter, provide fundamental information regarding changes in ventricular compliance and inotropy (the force of muscle contraction). These data are typically analyzed as pressure-volume (PV) loops. Whereas this technique is powerful, it requires a high level of expertise and is not as readily available as echocardiography. An elevation in the lung weight to body weight ratio can be a useful indication of pulmonary venous congestion, but care must be taken to remove and weigh the lungs rapidly after sacrifice of the mouse to avoid postmortem edema of the lungs. Changes in circulating TNF $\alpha$ may be measured in serum or plasma using commercially available ELISA kits.

\subsection{Assessing changes in ventricular morphology}

Changes in cardiac mass are reported as total heart weight or LV weight normalized to body weight or tibia length. It is often important to report both, as differences in body weight due to fat content or edema often occur. Changes in morphology can be viewed in histological 4chamber views, and the presence of fibrosis visualized using trichrome (or picrosirius red) staining for collagen. Hypertrophy of individual cardiomyocytes is measured as changes in cell cross-sectional area. This technique requires careful controls to assure measurement of cardiomyocytes sectioned perpendicularly to the plane of evaluation. Care must also be taken to minimize shrinkage of the tissue during processing. Alternatively, individual cardiomyocytes can be enzymatically dissociated from the heart and the two dimensional surface area measured. This approach, however, is limited in the number of cells that can be analyzed, as well as the possibility of selection bias (e.g., differential survival) during cell isolation. Assessment of changes in fine structural detail, such as sarcomere disarray or the accumulation of autophagic vesicles, requires electron microscopy. When assessing any of these morphological parameters it is essential that the hearts be in a similar state of relaxation. This can be achieved by perfusing hearts first with PBS then Krebs-Henseleit solution (120 $\mathrm{m} M \mathrm{NaCl}, 4.5 \mathrm{~m} M \mathrm{KC} 1,2.5 \mathrm{~m} M \mathrm{CaCl}_{2}, 1.0 \mathrm{~m} M \mathrm{MgCl}_{2}, 27 \mathrm{~m} M \mathrm{NaHCO}_{3}, 1.0 \mathrm{~m} M$ $\mathrm{KH}_{2} \mathrm{PO}_{4}$, and $10 \mathrm{~m} M$ glucose, $\mathrm{pH}$ 7.4) prior to fixation.

\subsection{Assessing changes in gene expression and intracellular signaling cascades}

Cardiac hypertrophy and heart failure are associated with reactivation of a so-called fetal gene program in the ventricle. Many of these genes are expressed both during embryogenesis and in adult atria so it is essential to remove the atria before analysis of the LV. There is an increase in expression of the natriuretic (i.e., involved in the discharge of sodium through urine) peptides ANP (Nppa) and BNP (Nppb) as well as alpha skeletal actin (Acta l) and the fetal cardiac myosin heavy chain $\beta \mathrm{MHC}(M y h 7)$. There is a corresponding decrease in expression of the sarcoplasmic reticulum calcium ATPase SERCA2 (Atp2a2) and the adult cardiac myosin heavy chain $\alpha \mathrm{MHC}(M y h 6)$. Of course, many other changes in transcriptional profiles occur during cardiac remodeling, but changes in expression of these genes are widely used as markers of 
pathological remodeling. Sequences of several useful real-time PCR primers are provided in Table 17.1.

Activation of a variety of intracellular signaling cascades has been implicated in cardiac remodeling (Heineke and Molkentin, 2006); many of these cascades are also involved in the regulation of autophagy. Whereas these pathways are too numerous to detail here, several recent reviews are available which provide links to techniques for monitoring these pathways (Frey and Olson, 2003; Heineke and Molkentin. 2006) (see also the chapters by Chiacchiera and Simone and by Ikenoue et al., in volume 452). Many of these signaling cascades involve changes in phosphorylation that are rapidly reversed once the heart is removed and stops beating. Thus, when harvesting hearts to examine changes in protein phosphorylation, the heart should be freeze-clamped in liquid nitrogen immediately upon removal from the chest without taking time to weigh it or washing in either PBS or Krebs-Henseleit solution.

\subsection{Distinguishing between adaptive and maladaptive remodeling of the heart}

A striking feature of the heart is its capacity for structural remodeling in response to changes in environmental demand (Hill and Olson, 2008), Indeed, the heart is a remarkably plastic organ, capable of growing or shrinking in the setting of a variety of physiological or pathological stimuli. In some instances, cardiac growth is beneficial, facilitating the organism's response to exercise, postnatal development, or pregnancy. In other instances, such as the stress of hypertensive disease or infarction, cardiac remodeling is maladaptive, predisposing to arrhythmia and heart failure. In yet other instances, such as prolonged bed rest or weightlessness, the heart shrinks.

It is impossible to distinguish between pathological and physiological heart growth on the basis of heart weight to body weight ratios alone. Pathological hypertrophy can occur without any overt clinical signs or measurable changes in cardiac function. This is where analysis of fetal gene expression and the shift in myosin heavy chain isoform from $\alpha \mathrm{MHC}$ to $\beta \mathrm{MHC}$ is extremely useful. For instance, mice subjected to a month of exercise training may have an increase in heart weight body weight ratio comparable to that elicited by TAB surgery. Neither heart manifests a decrease in cardiac function or elevated TNF $\alpha$ levels, such as occurs in mice subjected to STAB. However, fetal gene expression and $\beta$ MHC protein levels are increased in the TAB heart and not in the exercise-induced hypertrophic heart. Thus, it is critical to analyze a full range of parameters, from changes in transcription to functional clinical assessments, to distinguish between adaptive and maladaptive hypertrophy.

\section{IN VITRO MODELS OF LOAD-INDUCED HYPERTROPHY}

It is not possible to model load-induced hypertrophy in cultured cells. However, cultured ventricular myocytes isolated from neonatal rat pups (NRVM) are the standard model for studying the biology of cardiac myocytes in culture. We include protocols for examining autophagy in NRVM, because they can be used to test the direct effect of downstream mediators, such as TNF $\alpha$, on autophagic processes in culture. Detailed procedures for their isolation and culture have been published (Maass and Buvoli. 2007).

\section{TECHNIQUES TO ANALYZE CARDIOMYOCYTE AUTOPHAGY}

The heart poses a number of unique challenges for analyzing autophagy. Although hypertrophy involves an increase in the size of cardiomyocytes, the heart is composed of many other cell types including fibroblasts, endothelial cells, smooth muscle cells and leukocytes. Indeed, it is estimated that these other cell types account for between $40 \%$ and $60 \%$ of the cell population in the heart (Brown et al., 2005), although the bulk mass of protein is contributed by cardiomyocytes. Here, we describe cardiomyocyte-specific GFP-LC3 reporter mice we have 
developed that can be used to monitor autophagic activity specifically in cardiomyocytes. The presence of a densely packed sarcomere also poses unique challenges for immunohistochemistry and for isolation of soluble and insoluble proteins from heart. We outline some of the techniques that have proven useful.

\subsection{The aMHC-GFP-LC3 cardiomyocyte-specific autophagy reporter mouse}

GFP fused to microtubule-associated protein 1 light chain 3 (LC3), the mammalian homologue of yeast Atg8, provides a useful tool for monitoring autophagic activity in vivo. LC3 is an intermembrane component of the early autophagosome, and its redistribution from a diffuse cytosolic signal to punctate dots is a sensitive and specific indicator of autophagy (Kabeya et al., 2000). Transgenic mice carrying a GFP-LC3 transgene driven by a constitutive CAG-CMV promoter/enhancer have proven to be a powerful tool for in vivo analysis of autophagy (Mizushima et al., 2004), where autophagosome abundance can be measured as an increase in GFP-LC3 puncta using fluorescence microscopy. Expression of the CAGpCMV-GFP-LC3 transgene does not increase the level of autophagic activity. In these mice, however, the GFPLC3 fusion protein is expressed in all cell types. Therefore, to study autophagy specifically in cardiomyocytes, we used the alpha myosin heavy chain $(\alpha \mathrm{MHC})$ promoter to drive cardiomyocyte-specific expression of a GFP-LC3 transgene in the adult heart. (The $\alpha M H C$ $G F P-L C 3$ transgene is not expressed during embryogenesis, so it cannot be used to study the role of autophagy during cardiac development.) Two independent lines of $\alpha M H C-G F P-L C 3$ transgenic mice were obtained. Neither line shows any changes in heart development, cardiac growth, or cardiac function when compared to wild-type littermates. The $\alpha M H C-G F P-L C 3$ transgene can be easily identified by PCR using the forward and reverse primers $\left(5^{\prime}\right.$ CATCGAGCTGAAGGGCATCG-3' and 5'-CTATAATCACTGGGATCTTGGTG-3'). These reporter mice can be subjected to TAB and sTAB stress as well as mated to genetic backgrounds or transgenic mice of interest.

\subsection{Detection of GFP-positive autophagic vesicles in the aMHC-GFP-LC3 reporter mouse}

1. Retrograde perfuse heart with ice-cold PBS ( $10 \mathrm{~mL}$ for an adult mouse).

2. Perfuse with freshly prepared ice-cold $4 \%$ paraformaldehyde (PFA)/PBS (15-20 mL for an adult mouse).

3. Remove the heart and rinse with PBS.

4. Fix in $4 \%$ PFA/PBS for $4 \mathrm{~h}$ at $4{ }^{\circ} \mathrm{C}$.

5. Section the heart in half for a 4-chamber view.

6. Equilibrate the heart in $30 \%$ sucrose/PBS overnight at $4{ }^{\circ} \mathrm{C}$.

7. Mount the sucrose-equilibrated hearts in freezing matrix (TFM, Triangle Bioscience, Raleigh, NC, catalog number: TFM-C).

8. Section with a cryostat ( $8 \mu \mathrm{m}$, Leica CM3000) and mount on glass slides.

9. Air-dry the slides (the sections can be stored at $-80{ }^{\circ} \mathrm{C}$ until use).

10. Rinse slides with PBS for 5 min.

11. Mount slides with Vectashield (Vector Laboratories, Burlingame, CA, catalog number: H1000) mounting media and a coverslip.

12. Cover-slipped slides can be store at $-20^{\circ} \mathrm{C}$ until photographing.

13. The GFP signal can be viewed using an Endow GFP Bandpass Emission filter set (Chroma 41017; Rockingham, VT). 


\section{Notes}

1. Results can be expressed as the number of GFP-LC3 dots per field of high magnification or the number of GFP-LC3 dots per cell. Because the size of individual cardiomyocytes increases with hypertrophy, we suggest expressing the results as the number of GFP-LC3 dots per field of high magnification.

2. Multiple fields should be photographed and quantified. Autophagic activity is not homogeneous across the heart, neither is transgene expression driven by the $\alpha \mathrm{MHC}$ promoter. For unclear reasons, autophagy activity is greater in the basal septum both at rest and in response to starvation or pressure overload (Zhu et al., 2007). Therefore it is important to quantify different regions of the heart independently.

3. The heart has a high level of autofluorescence. For photographic images, a Texas Red filter set (red) can be used to exclude the autofluorescent signal.

\section{IMMUNOHISTOCHEMISTRY FOR LAMP-1 OR CATHEPSIN D TO MONITOR CHANGES IN LYSOSOMAL ABUNDANCE}

Quantification of autophagosome abundance is insufficient to study autophagy, a flux pathway of protein and organelle recycling. (Increases in autophagosome abundance could derive simply from defects in the pathway downstream, like a traffic tie-up occurring due to blockage of the road in the distance.) Thus, it is critical to evaluate autophagy using a variety of strategies and to test downstream mechanisms of autophagosome processing.

Autophagosomes fuse with lysosomes to form autolysosomes in which the cargo within in the autophagosome is degraded. To evaluate possible changes in the processing of autophagosomes, it is important to monitor changes in lysosome abundance. This section describes the procedure for immunohistochemical detection of the lysosome-associated membrane protein 1 (LAMP-1) and the lysosomal protease cathepsin D in the heart. Immunohistochemisty of striated muscle can be difficult because of the abundance of sarcomeric proteins. Because these proteins represent such a high percentage of the total protein present in a cardiomyocyte, a low affinity cross-reaction of an antibody to a sarcomeric protein could result in a non-specific banded sarcomeric pattern of staining. We have established reliable protocols for LAMP-1 and cathepsin D staining in the heart.

\subsection{Immunohistochemistry for LAMP-1 or cathepsin D}

1. Perfuse and fix hearts as for GFP-LC3 (steps 1-3 previously).

2. Fix in $4 \%$ PFA/PBS overnight at $4{ }^{\circ} \mathrm{C}$.

3. Dehydrate and paraffin embed: Take tissue through a series of alcohol dehydration steps at room temperature ( $70 \%$ for 30 seconds, followed by $80 \%, 90 \%, 96 \%$, and $100 \%$ each for an hour) followed by 3 complete changes of xylene (i.e., the xylene is removed and replaced every hour for a total of 3 times). The samples are then immersed in Paraplast Plus (McCormick Scientific catalog number 502004) for $1 \mathrm{~h}$ $10 \mathrm{~min}$. Then, the Paraplast Plus is removed and replaced with fresh Paraplast Plus for $1 \mathrm{~h} 20 \mathrm{~min}$, and the process is repeated once more for $1 \mathrm{~h} 30 \mathrm{~min}$. The embedding in paraffin wax should be performed at $60^{\circ} \mathrm{C}$ with agitation and under vacuum.

4. Section with microtome for 4-chamber view ( $5 \mu \mathrm{m}$, Leica RM2255 rotary microtome), slides may be stored at room temperature until needed.

5. Bake slides in $56{ }^{\circ} \mathrm{C}$ oven for $1 \mathrm{~h}$.

6. Dewax sections in xylene for 2 changes, 5 min each. 
7. Remove xylene from sections by washing in $100 \%$ ethanol, 3 changes, 2 min each.

8. Hydrate sections through 3 changes of xylene ( 5 min each), 3 changes of $100 \%$ ethanol (1.5 min each), into $95 \%$ ethanol (once for $1.5 \mathrm{~min}$ ), and then into water.

9. Wash in $1 \mathrm{x}$ PBS for $5 \mathrm{~min}$.

10. Fix in $4 \%$ PFA for $10 \mathrm{~min}$.

11. Wash 3 times in PBS, 5 min for each wash.

12. Outline sections with PAPPEN (Invitrogen, Carlsbad, CA, catalog number 00-8877).

13. Permeabilize sections in $0.3 \%$ Triton $X-100$ in PBS for 10 min.

14. Wash three times in PBS, 5 min for each wash.

15. Incubate in blocking buffer (1xPBS, $3 \%$ goat serum (Vector Biolabs, S1000), $0.5 \%$ BSA (Fisher Scientific, BP6755)) for $1 \mathrm{~h}$.

16. Incubate with rat anti-LAMP-1 (1:500 dilution in blocking buffer; Santa Cruz Biotechnology, SC-19992) or rabbit anti-cathepsin D (1:100 dilution; Santa Cruz Biotechnology, SC-10725) in blocking buffer overnight at $4{ }^{\circ} \mathrm{C}$ in a humidified environment.

17. Wash 3 times in PBS, 5 min for each wash.

18. Incubate with $\mathrm{Cy} 3$ conjugated donkey anti-rat $\operatorname{IgG}$ (1:100 dilution in blocking buffer; Jackson Immunology, 712-166-153) or donkey anti-rabbit IgG (1:100 dilution in blocking buffer; Jackson Immunology, 711-165-152) for $30 \mathrm{~mm}$.

19. Wash 3 times in PBS, 5 min each wash.

20. Mount with Vectashield antifade mounting medium (Vector Laboratories, Burlingame, CA, catalog number: $\mathrm{H} 1000)$; slides may be stored at $-20^{\circ} \mathrm{C}$ until viewing.

21. View using a Cy3 filter set (Chroma 41007; Rockingham, VT).

\subsection{Immunohistochemistry for ubiquitinated aggregates}

Severe pressure overload triggers accumulation of aggregates of polyubiquitinated protein in the perinuclear zone of the cardiomyocyte (Rothermel and Hill, 2008; Tannous et al., 2008b). Immunohistochemical imaging of the aggregates is similar to the procedure described previously but requires addition of a microwave-mediated antigen retrieval step and an additional blocking step, because of the use of a mouse monoclonal anti-ubiquitin antibody on mouse tissue sections. The microwave-mediated antigen retrieval step involves microwaving the sample in acidic citrate-based buffer, a process that breaks protein-protein aldehyde crosslinks initially established by tissue fixation. With cleavage of these aldehyde bonds, antibodies are able to access more of their specific antigenic epitopes and anneal with affinity otherwise unachievable.

1. Perfuse, fix, embed, and section as previously (steps 1-4).

2. Air-dry slides.

3. Dewax sections in xylenc for two changes, 5 nun each.

4. Remove xylene from sections in $100 \%$ ethanol for 3 changes, 2 min each.

5. Hydrate sections through graded ethanol concentrations to $\mathrm{H}_{2} \mathrm{O}$ as described earlier. 
6. Perform microwave-induced epitope retrieval using lxBiogenex Citra (Biogenex, San Ramon, CA, HK0869K) 10 min at $95^{\circ} \mathrm{C}$.

7. Cool slides with partial change-out of $\mathrm{H}_{2} \mathrm{O}$ for $20 \mathrm{~min}$, where partial change-out means pouring out $\{1 / 3\}$ of the water and replacing the volume with room temperature water, thus gradually cooling down the water and the slides.

8. Wash in PBS 3 times, for 2 min each.

9. Permeabilize sections with $0.3 \%$ Triton $\mathrm{X}-100 / \mathrm{PBS}$ for $10 \mathrm{~mm}$.

10. Wash in PBS 3 times, for 2 min each.

11. Quench autofluorescence with $100 \mathrm{~m} M$ glycine (not $\mathrm{pH}$ adjusted) in PBS twice, for 8 min each.

12. Wash in PBS 3 times, for 2 min each.

13. Block endogenous mouse immunoglobulin activity by applying Mouse-on-Mouse (MOM) IgG Blocking Reagent diluted 1:27 in PBS and incubating for $1 \mathrm{~h}$ (Vector Laboratories, Burlingame, CA, catalog number BMK2202).

14. Wash in PBS 3 times, for 2 min each.

15. Equilibrate antigenic epitopes in MOM diluent by applying MOM Protein Concentrate (see previously) diluted in 1:12.5 m PBS and incubating for $5 \mathrm{~min}$.

16. Decant excess MOM diluent, wipe, and apply anti-ubiquitin (UB-1) primary antibody (Santa Cruz, catalog number sc-6085) diluted 1:400 in MOM diluent/PBS and incubate overnight at $4{ }^{\circ} \mathrm{C}$.

17. Wash in PBS 3 times, for 2 min each.

18. Apply MOM biotinylated anti-mouse IgG (see previously) diluted 1:250 in MOM diluent/PBS and incubate $10 \mathrm{~min}$.

19. Wash in PBS 3 times, for $2 \mathrm{~mm}$ each.

20. Apply fluorescein-avidin DCS (Vector Laboratories, Burlingame, CA, catalog number A2011) diluted 1:62 in PBS and incubate for $5 \mathrm{~min}$.

21. Wash in PBS 3 times, for 2 min each.

22. Rinse in $\mathrm{H}_{2} \mathrm{O}$ twice, for 5 min each.

23. Mask muscle specific autofluorescence with $0.1 \%$ Sudan black (Sigma-Aldrich; St. Louis, MO, catalog number S4261)/70\% ethanol for $30 \mathrm{~min}$ (Baschong et al., 2001).

24. Rinse in $\mathrm{H}_{2} \mathrm{O}$ twice, for 5 min each.

25. Mount with a coverslip with Vectashield.

\subsection{Immunohistochemistry of neonatal heart ventricular myocytes}

We use the following protocol to image alpha-B crystallin (Cry $\mathrm{AB})$ and desmin localized to protein aggregates in cultured cardiomyocytes (Tannous et al., 2008a). Neonatal heart ventricular myocytes (NRVM) should be grown on coverslips coated with laminin (Fisher Scientific 08774385). PBS used in this procedure should contain $\mathrm{Ca}^{2+}$ and $\mathrm{Mg}^{2+}$ ("PBS+") to maintain cell integrity. This can be obtained commercially or made using the recipe supplied here:

1 Rinse cells with PBS+ for 5 min, 3 times at room temperature (RT).

2 Fix cells with $4 \%$ PFA/PBS+ for $5 \mathrm{~mm}$ on ice. 
3 Rinse cells with PBS for 5 min 3 times at RT.

4 Permeabilize cells with $0.1 \%$ Triton/PBS for 2 min at RT.

5 Block nonspecific binding with 1.0\% BSA/PBS for $15 \mathrm{~min}$ at RT.

6 Incubate cells, coverslip face down, on labeled microscope slides with $50 \mu \mathrm{L}$ of primary antibody/1\%BSA/PBS in a humid chamber for $1 \mathrm{~h}$ at RT. A range of antibody dilutions should be tested initially. The concentration of antibody usually needs to be much higher than that used for Western blot analysis. To label concurrently for desmin and CryAB, use mouse anti-desmin (1:50; Abcam, catalog number AB8592) and rabbit anti-CryAB (1:50; Vision, catalog number Abcrys512). Remember to include a PBS control without antibody.

7 Float coverslip off slide with $1.0 \% \mathrm{BSA} / \mathrm{PBS}$, and using forceps, carefully return to a 6-well plate, keeping the orientation intact.

8 Rinse cells with PBS for 5 min 3 times at RT.

All remaining steps to be conducted in subdued lighting:

9 Incubate cells, coverslip face down, on labeled microscope slides with $50 \mu \mathrm{L}$ of 1:12 diluted fluorescent secondary antibodies/ 1.0\%BSA/PBS in a humid chamber for 30 min at RT. Use rhodamine-tagged goat anti-mouse IgG (Jackson ImmunoResearch, catalog number 115-025-003) to detect desmin and fluorescein-tagged goat antirabbit (Jackson Immuno Research, catalog number 111-095-045) to detect CryAB.

10 Float coverslip off slide with $1.0 \%$ BSA/PBS, and using forceps, carefully return to 6 -well plate, keeping the orientation intact.

11 Rinse the coverslips with $1.0 \% \mathrm{BSA} / \mathrm{PBS}$ for $2 \mathrm{~min}$.

12 Stain cells with Hoechst 33258 (Molecular Probes, catalog number H3570) diluted 1:50 in PBS for 2 min at RT.

13 Rinse cells with PBS for 2 min 3 times at RT.

14 Rinse cells with distilled water for 2 min twice at RT.

15 Mount coverslips on microscope slides with $\sim 18 \mu \mathrm{L}$ Vectashield mounting medium.

\subsection{Preparation of PBS with $\mathrm{Ca}^{2+}$ and $\mathrm{Mg}^{2+}(\mathrm{PBS}+)$}

To avoid precipitation, two 20x stock solutions should be prepared and autoclaved separately.

\begin{tabular}{ll}
\hline solution A : & $348 \mathrm{~m} M \mathrm{Na}_{2} \mathrm{HPO}_{4}: 46.86 \mathrm{~g}$ \\
& $70 \mathrm{~m} M \mathrm{NaH}_{2} \mathrm{PO}_{4}: 9.66 \mathrm{~g}$ \\
solution B : & Bring to 1 liter and autoclave \\
& $18 \mathrm{~m} M \mathrm{CaCl}_{2}: 2.6 \mathrm{~g}$ \\
& $70 \mathrm{~m} M \mathrm{KCl}^{2}: 5.2 \mathrm{~g}$ \\
& $18 \mathrm{~m} M \mathrm{MgCl}_{2}: 3.6 \mathrm{~g}$ \\
& $2.74 M \mathrm{NaCl}: 160 \mathrm{~g}$ \\
& Bring to 1 liter and autoclave
\end{tabular}

Store stocks at room temperature. Add $5 \mathrm{~mL}$ of each to $90 \mathrm{~mL}$ of water for working stock. Do not refrigerate.

\subsection{Generation of a rabbit polyclonal anti-LC3 antibody}

One of the initial steps in autophagy is conversion of LC3 from the cytosolic LC3-I isoform to the PE-conjugated membrane-bound LC3-II isoform. Changes in the abundance of LC3-II or the ratio of LC3-II to LC3-II + LC3-I have been used to track changes in autophagic flux 
(though the latter is discouraged because of differences in the amount of LC3-I in different cells/tissues and the detection of this form by many antibodies). Although commercial antibodies are available, we have had limited success using these on western analysis of heart extracts. We therefore generated our own affinity-purified antipeptide antibody. Briefly, ammo acids $2-15$ from the N-terminus of murine LC3 (PSEKTFKQRRSFEQC) were chemically coupled to keyhole limpet hemocyanin (KLH). New Zealand white rabbits were used to generate antisera to the LC3-KLH conjugate, and specific antibodies were affinity purified using the same peptide. This antibody works well for Western blot analysis but not immunohistochemistry.

\section{ISOLATION OF LC3 PROTEINS FROM NRVM IN CULTURE}

1. Aspirate the medium and wash cells (approximately 1.2 million) twice with ice-cold PBS.

2. Add RIPA buffer $(150 \mathrm{~m} M \mathrm{NaCl}, 50 \mathrm{~m} M$ Tris-HCl, $\mathrm{pH} 7.4,1 \mathrm{~m} M$ EDTA, $1 \%$ Triton $\mathrm{X}-100,1 \%$ sodium deoxycholate, $0.1 \%$ SDS, plus protease inhibitors (Complete Mini-EDTA free, Roche 1836145) and scrape cells.

3. Transfer to a $1.5-\mathrm{mL}$ microcentrifuge tube and incubate on ice, agitating every $2-3$ $\min$ for $15 \mathrm{~min}$.

4. Centrifuge at $16,000 \mathrm{Xg}$ for $10 \mathrm{~min}$.

5. Transfer the supernatant fraction to a fresh $1.5-\mathrm{mL}$ microcentrifuge tube.

6. Quantify protein using coomassie blue reagent.

Note: Be sure to dilute protein extract in PBS to bring buffer components within the linear range of the protein assay being used.

\section{ISOLATION OF LC3 PROTEIN FROM HEART OR SKELETAL MUSCLE TISSUE}

Detergent extraction of some proteins from striated muscle can be difficult presumably because they become trapped in the tightly packed sarcomere, which becomes an insoluble mass upon addition of most detergents. Extraction of cardiac myosins is carried out by homogenization in a buffer lacking detergent followed by incubation on ice. We have developed a similar technique for extracting LC3.

1. Use a $2-\mathrm{mL}$ glass Dounce homogenizer to homogenize finely minced pieces of left ventricle in lysis buffer $(150 \mathrm{mM} \mathrm{NaCl}, 20 \mathrm{~m} M$ Tris-HCl, pH7.4, $10 \mathrm{~m} M$ EDTA plus protease inhibitors) on ice using both the $\mathrm{A}$ and $\mathrm{B}$ pestles.

2. Centrifuge at $500 \mathrm{Xg}$ for $10 \mathrm{~min}$ at $4{ }^{\circ} \mathrm{C}$.

3. Transfer the supernatant fraction to a fresh microcentrifuge tube.

4. Quantify the protein using coomassie reagent.

5. Add an equal volume of $2 x$ SDS sample buffer to the supernatant fraction.

Note: Components in the low-speed supernatant may settle with time. Be sure to resuspend before removing a sample for protein determination. If the extract is viscous due to the presence of genomic DNA, it can be passed through glass wool prior to loading for SDS-PAGE.

\subsection{Western blot analysis using the LC3 antibody}

1. Separate proteins on a $12.5 \%$ SDS-PAGE gel. 
2. Transfer protein to a nitrocellulose membrane at $25 \mathrm{~V}$ overnight at $4{ }^{\circ} \mathrm{C}$.

3. Incubate membrane with blocking buffer (5\% milk, 1xTBS, $0.1 \%$ Tween 20$)$ for $1 \mathrm{~h}$ at RT.

4. Incubate with anti-LC3 polyclonal antibody (1:2,000 dilution) in blocking buffer overnight at $4{ }^{\circ} \mathrm{C}$ or at RT for $1 \mathrm{~h}$.

5. Wash in TBST (lxTBS, 0.1\% Tween 20) for 10 min 4 times.

6. Incubate with HRP-conjugated goat antirabbit secondary antibody (1:5000 dilution) in blocking buffer for $1 \mathrm{~h}$ at RT.

7. Wash in TBST for 10 min 4 times.

8. Incubate with HRP substrate solutions (e.g., ECL PLUS).

\subsection{Monitoring levels of other autophagy-related proteins}

A change in the level of several other autophagy-related proteins may indicate altered flux through autophagic pathways. Beclin 1, the mammalian homolog of yeast Atg6 (Liang et al., 1999), binds to class III phosphoinositides-3-kinase (PI3K) and recruits other autophagy molecules to the membrane at an early stage of autophagosome formation (Kibara et al., 2001). In sTAB hearts, an increase in autophagy is accompanied by an increase in Beclin 1 protein abundance (Zhu et al., 2007). Cardiomyocyte-restricted forced overexpression of the gene coding for Beclin 1 ( $\alpha M H C$-beclin 1) increases stress-induced cardiac autophagy (Zhu et al., 2007). Conversely, autophagy is diminished in the hearts of heterozygous beclin $1^{+/-}$ mice (Zhu et al., 2007). It is important to be aware that Beclin 1 expression does not always increase when autophagy is activated. For instance, during starvation-induced autophagy, Beclin 1 levels in the heart do not change (Zhu et al., 2007). Furthermore, changes in Beclin 1 levels do not necessarily correlate with changes in processing of LC3-I (Matsui et al., 2007).

Polyubiqitinated aggregates of misfolded or damaged proteins can be degraded via autophagy. A protein named p62, also called SQSTM1, binds both to LC3 and polyubiquitin and is thought to be involved in this process (Pankiv et al., 2007). Consistent with this, disruption of autophagic activity in cardiomyocytes leads to accumulation of poly-ubiquitinated proteins and p62 (Nakai et al., 2007; Tannous et al., 2008b).

Western blot analysis of Beclin 1, p62, and protein ubiquitination can be carried out following the protocol described above for LC3. Incubate primary antibodies in blocking buffer overnight at $4{ }^{\circ} \mathrm{C}$. Monoclonal Beclin 1 antibody (BD Bioscience, catalog number 612113 ) is used at a 1:500 dilution. Monoclonal p62 antibody (BD Bioscience, catalog number 610832) is used at a 1:500 dilution, and the polyclonal ubiquitin antibody (Zymed for immunohistochemistry (131600); Dako for western blot, catalog number Z0458) is used at 1:500 dilution.

\subsection{Separation of soluble proteins from insoluble aggregates}

Protein aggregation and increased autophagic activity are common features of many neurodegenerative disorders (Rubinsztein, 2006). We have observed an association between the accumulation of protein aggregates and increased autophagic activity in both the pressure overloaded heart (Tannous et al., 2008b) and in a genetic model of desmin-related cardiomyopathy (Rothermel and Hill. 2008; Tannous et al., 2008a). Protein aggregates can be both soluble and insoluble, and there is mounting evidence that the soluble preaggregate is the more toxic of the species (Arrasate et al., 2004; Rubinsztein, 2006). The two protein extraction methods provided above for NRVM and heart were optimized for tracking LC3 and are not appropriate for analyzing soluble and insoluble proteins. In the case of the NRVM extraction, 
both soluble and insoluble proteins are present, whereas in the heart extraction, insoluble protein aggregates would be discarded. Thus, the following extractions are designed to separate soluble and detergent insoluble proteins. We have listed $1 \%$ Triton X-100 as the detergent for NRVM and 1\% NP-40 in the heart extraction, however, the two give similar results in either protocol.

\section{SOLUBLE/INSOLUBLE FRACTIONATION OF NRVM}

1. Harvest cells by digestion in trypsin $(0.25 \%$ dissolved in Hanks' Balanced Salt Solution containing $2.21 \mathrm{mM}$ EDTA) at $37^{\circ} \mathrm{C}$ for $\sim 5 \mathrm{~min}$. Pool 3 wells (from a 6 wellplate) to make 1 sample. Centrifuge at $3000 \mathrm{Xg}, 5 \mathrm{~min}$.

2. Aspirate off the trypsin. Wash cells once with PBS, centrifuging for 5 min at 3000 $\mathrm{X} g$.

3. Aspirate off the PBS. Resuspend the cell pellet in $200 \mu \mathrm{L}$ of Triton Buffer $(10 \mathrm{~m} M$ Tris, $150 \mathrm{~m} M \mathrm{NaCl}, 1 \mathrm{~m} M$ EDTA, $1 \%$ Triton $X-100$, protease and phosphatase inhibitors (Complete Mini-EDTA free, Roche 1836145). Pass the lysate through an insulin syringe (Fisher Scientific 1482679) 5 times. Incubate on ice for (at least) 15 $\min$.

4. Centrifuge at $12,000 \mathrm{Xg}, 15 \mathrm{~min}$, at $4{ }^{\circ} \mathrm{C}$.

5. Collect the supernatant fraction as the soluble fraction. Wash the pellet fractions 3 times with cold PBS (add $1 \mathrm{~mL}$ PBS to each pellet, centrifuge at 12,000Xg for 10 min). Try not to disturb the pellet during each wash.

6. Resuspend the washed pellet in $200 \mu \mathrm{L}$ of $1 \mathrm{xSDS}$ loading buffer (dilute $2 \mathrm{xSDS}$ loading buffer in PBS).

\subsection{Soluble/insoluble fractionation from heart or skeletal muscle}

As discussed earlier, protein extraction from heart tissue can be difficult, because the sarcomere becomes insoluble when the tissue is homogenized in the presence of detergent. We have overcome this problem by first solubilizing myosins in a detergent-free extraction buffer and then adding detergent.

1. Dounce homogenize finely minced heart tissue in $500 \mu \mathrm{L}$ to $1 \mathrm{~mL}$ (depending on size of tissue) of homogenization buffer $\left(0.3 M \mathrm{KC} 1,0.1 M \mathrm{KH}_{2} \mathrm{PO}_{4}, 50 \mathrm{mM} \mathrm{K} \mathrm{HPO}_{4}, 10\right.$ $\mathrm{m} M$ EDTA, $4 \mathrm{~m} M$ Na orthovanadate, $100 \mathrm{~m} M \mathrm{NaF}$, plus protease inhibitors).

2. Pass the homogenized sample through 100 micron mesh (Tetko, catalog number AC38842) on ice, collecting the lysate run-through.

3. Incubate the lysate on ice for $30 \mathrm{~min}$.

4. Transfer a portion of the sample to another microcentrifuge tube, and add 10\% NP-40 (Sigma, catalog number 18896) to achieve a final concentration of $1 \%$ NP-40. Incubate on ice for 15-30 min.

5. Centrifuge at $13,000 \mathrm{rpm}$ for $15 \mathrm{~min}, 4^{\circ} \mathrm{C}$.

6. Collect the supernatant fraction as the soluble fraction (contains sarcomere).

7. With the pellets, wash 3 times with cold PBS (add $1 \mathrm{~mL}$ PBS to each pellet, centrifuge at $13,000 \mathrm{rpm}$ for $10 \mathrm{~min}$ ).

8. Resuspend the washed pellet in $1 \%$ SDS, $10 \mathrm{~m} M$ Tris buffer, $\mathrm{pH} 8.0$. 
Note: Tissue disruption with a Dounce homogenizer is essential for this procedure, as it ensures that all cells are broken open despite the lack of detergent. The soluble fraction will contain all of the sarcomeric proteins and is therefore ideal for monitoring shifts in $\alpha \mathrm{MHC}$ and $\beta \mathrm{MHC}$ content by Western blot analysis.

\section{PERSPECTIVE}

Accumulating evidence links autophagy with the pathogenesis of multiple forms of heart disease, suggesting that autophagy may be a novel target of therapy. However, we and others have shown that the regulation and contribution of autophagy to heart disease is dose and context dependent, which poses new questions and special challenges (Rothermel and Hill, 2007). We believe that the mouse models of heart failure we have established and the methods we developed for monitoring autophagy in heart will be useful in dissecting the precise role(s) of cardiac autophagy in multiple forms of heart disease. Ultimately, it is our hope that insights gleaned using these tools will lead to the novel strategies of therapy for these devastating diseases.

\section{Acknowledgments}

This work was supported by grants from the Donald W. Reynolds Cardiovascular Clinical Research Center (JAH), NIH (BL-72016, BAR; HL-075173, JAH; HL-080144, JAH), and AHA (0655202Y, BAR; 06400S4N, JAH).

\section{REFERENCES}

Arrasate M, Mitra S, Schweitzer ES, Segal MR, Finkbeiner S. Inclusion body formation reduces levels of mutant huntingtin and the risk of neuronal death. Nature 2004;431:805-810. [PubMed: 15483602]

Baschong W, Suetterlin R, Laeng RH. Control of autofluorescence of archival formaldehyde-fixed, paraffin-embedded tissue in confocal laser scanning microscopy (CLSM). J. Histochem. Cytochem 2001;49:1565-1572. [PubMed: 11724904]

Brown RD, Ambler SK, Mitchell MD, Long CS. The cardiac fibroblast: Therapeutic target in myocardial remodeling and failure. Annu. Rev. Pharmacol. Toxicol 2005;45:657-687. [PubMed: 15822192]

Dammrich J, Pfeifer U. Cardiac hypertrophy in rats after supravalvular aortic constriction. II. Inhibition of cellular autophagy in hypertrophying cardiomyocytes. Virchows. Arch B. Cell Pathol. Incl. Mol. Pathol 1983;43:287-307. [PubMed: 6137901]

Decker RS, Decker ML, Herring GH, Morton PC, Wildenthal K. Lysosomal vacuolar apparatus of cardiac myocytes in heart of starved and refed rabbits. J. Mol. Cell Cardiol 1980;12:1175-1189. [PubMed: 7441766]

Decker RS, Wildenthal K. Lysosomal alterations in hypoxic and reoxygenated hearts 1. Ultrastructural and cytochemical changes. American Journal of Pathology (98) 1980:425-444. [PubMed: 7355988]

Elsasser A, Vogt AM, Nef H, Kostin S, Mollmann H, Skwara W, Bode C, Hamm C, Schaper J. Human hibernating myocardium is jeopardized by apoptotic and autophagic cell death. J. Am. Cell. Cardiol 2004;43:2191-2199.

Frey N, Katus HA, Olson EN, Hill JA. Hypertrophy of the heart: A new therapeutic target? Circulation 2004;109:1580-1589. [PubMed: 15066961]

Frey N, Olson EN. Cardiac hypertrophy: The good, the bad, and the ugly. Annu. Rev. Physiol 2003;65:4579. [PubMed: 12524460]

Hein S, Arnon E, Kostin S, Schonburg M, Elsasser A, Polyakova V, Bauer EP, Klovekorn WP, Schaper J. Progression from compensated hypertrophy to failure in the pressure-overloaded human heart: Structural deterioration and compensatory mechanisms. Circulation 2003;107:984-991. [PubMed: 12600911]

Heineke J, Molkentin JD. Regulation of cardiac hypertrophy by intracellular signalling pathways. Nat. Rev. Mol. Cell Biol 2006;7:589-600. [PubMed: 16936699] 
Hill JA, Karimi M, Kutschke W, Davisson RL, Zimmerman K, Wang Z, Kerber RE, Weiss RM. Cardiac hypertrophy is not a required compensatory response to short-term pressure overload. Circulation 2000;101:2863-2869. [PubMed: 10859294]

Hill JA, Olson EN. Cardiac plasticity. New. Engl. J. Med 2008;358:1370-1380. [PubMed: 18367740]

Kabeya Y, Mizushima N, Ueno T, Yamamoto A, Kirisako T, Noda T, Kominami E, Ohsumi Y, Yoshimori T. LC3, a mammalian homologue of yeast Apg8p, is localized in autophagosome membranes after processing. EMBO J 2000;19:5720-2728. [PubMed: 11060023]

Kihara A, Kabeya Y, Ohsumi Y, Yoshimori T. Beclin-phosphatidylinositol 3-kinase complex functions at the trans-Golgi network. EMBO Reports 2001;2:330-335. [PubMed: 11306555]

Klionsky DJ. Autophagy: From phenomenology to molecular understanding in less than a decade. Nat. Rev. Mol. Cell Biol 2007;8:931-937. [PubMed: 17712358]

Knaapen MWM, Davies MJ, De Bie M, Haven AJ, Martinet W, Kockx MM. Apoptotic versus autophagic cell death in heart failure. Cardiovascular Research 2001;51:304-312. [PubMed: 11470470]

Kostin S, Pool L, Elsasser A, Hein S, Drexler HC, Arnon E, Hayakawa Y, Zimmermann R, Bauer E, Klovekorn WP, et al. Myocytes die by multiple mechanisms in failing human hearts. Circ. Res 2003;92:715-724. [PubMed: 12649263]

Kundu M, Thompson CB. Autophagy: Basic principles and relevance to disease. Annu. Rev. Pathol 2008;3:427-455. [PubMed: 18039129]

Levine B, Kroemer G. Autophagy in the pathogenesis of disease. Cell 2008;132:27-42. [PubMed: 18191218]

Levy D, Larson MG, Vasan RS, Kannel WB, Ho KK. The progression from hypertension to congestive heart failure. JAMA 1996;275:1557-1562. [PubMed: 8622246]

Liang XH, Jackson S, Seaman M, Brown K, Kempkes B, Hibshoosh H, Levine B. Induction of autophagy and inhibition of tumorigenesis by beclin 1. Nature 1999;402:672-676. [PubMed: 10604474]

Lockshin RA, Zakeri Z. Caspase-independent cell deaths. Current Opinion in Cell Biology 2002;14:727733. [PubMed: 12473346]

Maass AH, Buvoli M. Cardiomyocyte preparation, culture, and gene transfer. Methods. Mol. Biol 2007;366:321-330. [PubMed: 17568133]

Matsui Y, Takagi H, Qu X, Abdellatif M, Sakoda H, Asano T, Levine B, Sadoshima J. Distinct roles of autophagy in the heart during ischemia and reperfusion: Roles of AMP-activated protein kinase and Beclin 1 in mediating autophagy. Circ. Res 2007;100:914-922. [PubMed: 17332429]

Mizushima N. Autophagy: Process and function. Genes Dev 2007;21:2861-2873. [PubMed: 18006683]

Mizushima N, Yamamoto A, Matsui M, Yoshimori T, Ohsumi Y. In vivo analysis of autophagy in response to nutrient starvation using transgenic mice expressing a fluorescent autophagosome marker. Mol. Biol. Cell 2004;15:1101-1111. [PubMed: 14699058]

Nakai A, Yamaguchi O, Takeda T, Higuchi Y, Hikoso S, Taniike M, Omiya S, Mizote I, Matsumura Y, Asahi M, Nishida K, Hori M, et al. The role of autophagy in cardiomyocytes in the basal state and in response to hemodynamic stress. Nat. Med 2007;13:619-624. [PubMed: 17450150]

Pankiv S, Clausen TH, Lamark T, Brech A, Bruun JA, Outzen H, Øvervatn A, Bjørkøy G, Johansen T. p62/SQSTM1 binds directly to Atg8/LC3 to facilitate degradation of ubiquitinated protein aggregates by autophagy. J. Biol. Chem 2007;282:24131-24145. [PubMed: 17580304]

Pfeifer U, Fohr J, Wilhelm W, Dammrich J. Short-term inhibition of cardiac cellular autophagy by isoproterenol. J. Mol. Cell Cardiol 1987;19:1179-1184. [PubMed: 3443985]

Rockman HA, Ross RS, Harris AN, Knowlton KU, Steinhelper ME, Field LJ, Ross J Jr. Chien KR. Segregation of atrial-specific and inducible expression of an atrial natriuretic factor transgene in an in vivo murine model of cardiac hypertrophy. Proc. Natl. Acad. Sci. USA 1991;88:8277-8281. [PubMed: 1832775]

Rosamond W, Flegal K, Furie K, Go A, Greenlund K, Haase N, Hailpern SM, Ho M, Howard V, Kissela $\mathrm{B}$, et al. Heart disease and stroke statistics-2008 update: A report from the American Heart Association Statistics Committee and Stroke Statistics Subcommittee. Circulation 2008;117:e25e146. [PubMed: 18086926]

Rothermel BA, Berenji K, Tannous P, Kutschke W, Dey A, Nolan B, Yoo KD, Demetroulis E, Gimbel M, Cabuay B, et al. Differential activation of stress-response signaling in load-induced cardiac hypertrophy and failure. Physiol. Genomics 2005;23:18-27. [PubMed: 16033866] 
Rothermel BA, Hill JA. Myocyte autophagy in heart disease: Friend or foe? Autophagy 2007;3:632-634. [PubMed: 17786025]

Rothermel BA, Hill JA. The heart of autophagy: Deconstructing cardiac proteotoxicity. Autophagy 2008;4(7):932-935. [PubMed: 18769158]

Rubinsztein DC. The roles of intracellular protein-degradation pathways in neurodegeneration. Nature 2006;443:780-786. [PubMed: 17051204]

Shimomura H, Terasaki F, Hayashi T, Kitaura Y, Isomura T, Suma H. Autophagic degeneration as a possible mechanism of myocardial cell death in dilated cardiomyopathy. Jpn. Circ. J 2001;65:965968. [PubMed: 11716248]

Shintani T, Klionsky DJ. Autophagy in health and disease: A double-edged sword. Science 2004;306:990-995. [PubMed: 15528435]

Sybers HD, Ingwall J, DeLuca M. Autophagy in cardiac myocytes. Recent. Adv. Stud. Cardiac. Struct. Metab 1967;12:453-463. [PubMed: 1032000]

Tannous P, Zhu H, Johnstone JL, Shelton JM, Rajasekaran NS, Benjamin IJ, Nguyen L, Gerard RD, Levine B, Rothermel BA, Hill JA. Autophagy is an adaptive response in desmin-related cardiomyopathy. Proc. Nail. Acad. Sci. USA 2008a;105:9745-9750.

Tannous P, Zhu H, Nemchenko A, Berry JM, Johnstone JL, Shelton JM, Miller PJ Jr. Rothermel BA, Hill JA. Intracellular protein aggregation is a proximal trigger of cardiomyocyte autophagy.

Circulation 2008b;117:3070-3078. [PubMed: 18541737]

Yamamoto S, Sawada K, Shimomura H, Kawamura K, James TN. On the nature of cell death during remodeling of hypertrophied human myocardium. J. Mol. Cell Cardiol 2000;32:161-175. [PubMed: 10652200]

Zhu H, Tannous P, Johnstone JL, Kong Y, Shelton JM, Richardson JA, Le V, Levine B, Rothermel BA, Hill JA. Cardiac autophagy is a maladaptive response to hemodynamic stress. J. Clin. Invest 2007;117:1782-1793. [PubMed: 17607355] 
